\title{
INVARIANTS OF LOCALLY CONFORMALLY FLAT MANIFOLDS
}

\author{
THOMAS BRANSON, PETER GILKEY, AND JUHA POHJANPELTO
}

\begin{abstract}
Let $M$ be a locally conformally flat manifold with metric $g$. Choose a local coordinate system on $M$ so $g=e^{2 h_{X}} d x \circ d x$ where $d x \circ d x$ is the Euclidean standard metric. A polynomial $P$ in the derivatives of $h$ with coefficients depending smoothly on $h$ is a local invariant for locally conformally flat structures if the expression $P\left(h_{X}\right)$ is independent of the choice of $X$. Form valued local invariants are defined similarly. In this paper, we study the properties of the associated de Rham complex. We show that any invariant form can be obtained from the previously studied local invariants of Riemannian structures by restriction. We show the cohomology of the de Rham complex of local invariants is trivial. We also obtain the following characterization of the Euler class. Suppose that for an invariant polynomial $P$, the integral $\int_{T^{m}} P\left|d v_{g}\right|$ vanishes for any locally conformally flat metric $g$ on the torus $T^{m}$. Then up to the divergence of an invariantly defined one form, the polynomial $P$ is a constant multiple of the Euler integrand.
\end{abstract}

\section{INTRODUCTION}

In the 1970 s, I. M. Singer posed the following question. Let $P(g)$ be an invariant of Riemannian metrics $g$ in $m$ dimensions which depends polynomially on the derivatives of the components of $g$ in any local coordinate system. Suppose that on any compact $m$ dimensional Riemannian manifold $M$ without boundary the integral

$$
\int_{M} P(g)\left|d v_{g}\right|
$$

is independent of the particular metric $g$ chosen. Here $\left|d v_{g}\right|$ is the smooth Riemannian measure associated to $g$; no orientability is assumed. Then does there exist a universal constant $c$ which is independent of $(M, g)$ so that

$$
\int_{M} P(g)\left|d v_{g}\right|=c \chi(M) ?
$$

This question was answered in the affirmative by E. Miller [12]. Gilkey [7] proved a local version of this result; if the conditions of Singer's question hold, then

$$
P=c E_{m}+\delta Q,
$$

Received by the editors March 15, 1994 and, in revised form, June 30, 1994.

1991 Mathematics Subject Classification. Primary 53A30, 53A55, 57R20.

Research of the second author was partially supported by the NSF (USA) and IHES (France). 
where $E_{m}$ is the Euler integrand, $Q$ is a one form valued invariant depending polynomially on the jets of the metric, and $\delta$ is the formal adjoint of the exterior derivative $d$. By Stokes' theorem and by the Gauss-Bonnet theorem, this local version implies the global (integrated) version. In [7], this result was extended from the class of polynomial invariants to the class of invariants with smooth dependence on jets of the metric up to a finite order. Note that if $m$ is odd, these results mean that $P$ is an exact divergence so that

$$
\int_{M} P(g)\left|d v_{g}\right| \equiv 0
$$

In this paper, we pose and answer a conformal analogue of Singer's question. Let $\circ$ by symmetrized tensor product. If $X$ is a system of local coordinates, let $d x \circ d x$ be the standard Euclidean metric. We say that $(M, g)$ is locally conformally flat if, for every point $P$ of $M$, there exists a system of local coordinates $X$ defined near $P$ and a smooth conformal factor $h_{X}$ so that

$$
g=e^{2 h_{x}} d x \circ d x \text { near } P \text {. }
$$

For example, any metric of constant sectional curvature is locally conformally flat. Since $M$ is always locally conformally flat if $m \leq 2$, we shall assume $m \geq 3$ in what follows. If $(M, g)$ is locally conformally flat, then the Pontrjagin forms vanish identically (see Avez [2]). Consequently, not every manifold admits a locally conformally flat metric; for example complex projective space $\mathbf{C} P^{n}$ for $n \geq 2$ admits no locally conformally flat metric.

Let $P(h)$ be a polynomial in the derivatives of $h$ with coefficients depending smoothly on $h$. If $g$ is locally conformally flat, we can choose a local coordinate system $X$ so that

$$
g=e^{2 h_{x}} d x \circ d x .
$$

Of course, there are many such coordinate systems; we say that $P$ is invariant if $P(g):=P\left(h_{X}\right)$ is independent of the choice of $X$ and depends only on the locally conformally flat metric $g$. Let $\mathscr{P}_{m, 0}$ be the space of such scalar invariants; we define the space of $n$ form valued polynomial invariants $\mathscr{P}_{m, n}$ similarly.

These are not invariants of the conformally flat structure; they are polynomials which are invariant under a suitable subgroup of the group of diffeomorphisms. A priori, it is not obvious that these invariants are the restriction of Riemannian invariants to this setting. Fortunately, it turns out that this is the case. Let $\rho_{i_{1} i_{2} i_{3} \ldots i_{\nu}}$ be the components of the symmetrized covariant derivatives of the Ricci tensor. Let $\mathscr{Q}_{m, n}$ be the space of $n$ form valued polynomial invariants in these variables. We contract indices in pairs to form scalar invariants; for example, the scalar curvature $\tau=\rho_{i i}$ is such an invariant. Form valued invariants are constructed similarly.

We introduce a grading based on counting the number of derivatives which appear; $\rho$ is homogeneous of degree 2 and each covariant derivative adds one to the degree. Let $\mathscr{P}_{m, n, p}$ and $\mathscr{Q}_{m, n, p}$ be the subspace of invariants which are homogeneous of degree $p$ in the jets of the metric. If $P$ is an $n$ form valued invariant polynomial, then $P$ is homogeneous of degree $p$ if and only if, for any positive constant $c$ and for any locally conformally flat metric $g$,

$$
P\left(c^{2} g\right)=c^{n-p} P(g) \text {. }
$$


This permits us to decompose

$$
\mathscr{P}_{m, n}=\bigoplus_{p} \mathscr{P}_{m, n, p} \quad \text { and } \quad \mathscr{Q}_{m, n}=\bigoplus_{p} \mathscr{Q}_{m, n, p}
$$

Theorem 0.1. (a) The forgetful functor is an isomorphism from $\mathscr{Q}_{m, n, p}$ to $\mathscr{P}_{m, n, p}$.

(b) If $n-p$ is odd or if $2 p<n$, then $\mathscr{P}_{m, n, p}=\{0\}$.

Remark. Since the Pontrjagin forms belong to $\mathscr{P}_{m, n, n}$, (b) gives another proof that the Pontrjagin forms vanish on locally conformally flat metrics. We also note that Robin Graham has proved that, on any Riemannian manifold, one can locally conformally change the metric to one in which all the symmetrized covariant derivatives of the Ricci tensor vanish; this is discussed by Lee and Parker [11].

Exterior differentiation $d$ and its dual, interior differentiation $\delta$, provide maps

$$
d: \mathscr{P}_{m, n, p} \rightarrow \mathscr{P}_{m, n+1, p+1} \text { and } \delta: \mathscr{P}_{m, n, p} \rightarrow \mathscr{P}_{m, n-1, p+1} .
$$

Let $X=\left(x_{1}, \ldots, x_{m}\right)$ be the standard periodic parameters on the torus $T^{m}$, and let $\mathscr{C}$ be the set of all metrics on $T^{m}$ giving the standard conformal structure; $g \in \mathscr{C}$ if and only if

$$
g=e^{2 h} d x \circ d x .
$$

Singer's conjecture is concerned with Riemannian invariants. It has an extension to the locally conformally flat category. In the following theorem, we will only need to require that $(0.1)$ vanishes for $g \in \mathscr{C}$ to conclude that $(0.2)$ holds for any locally conformally flat metric.

Theorem 0.2. Let $P \in \mathscr{P}_{m, 0}$ for $3 \leq m$. Assume that

$$
\int_{T^{m}} P(g)\left|d \nu_{g}\right|=0 \quad \forall g \in \mathscr{C} .
$$

Then there exists a constant $c$ and a 1-form $Q \in \mathscr{P}_{m, 1}$ so that

$$
P=c E_{m}+\delta Q \text {. }
$$

Consequently the following equation holds universally for any locally conformally flat metric $g$ on any compact $m$ dimensional manifold $M$ :

$$
\int_{M} P(g)\left|d v_{g}\right|=c \chi(M)
$$

There is a corresponding result for form valued invariants.

Theorem 0.3. Let $P \in \mathscr{P}_{m, n}$ for $1 \leq n \leq m$ and $3 \leq m$. If $n<m$, assume that $d P=0$. If $n=m$, assume that

$$
\int_{T^{m}} P(g)\left|d v_{g}\right|=0 \quad \forall g \in \mathscr{C} .
$$

Then there exists $Q \in \mathscr{P}_{m, n-1}$ such that $P=d Q$.

Remark. In Theorems 0.2 and 0.3 , the algebra of polynomial invariants may be replaced by the algebra of invariants with smooth dependence on the jets of the metric up to a finite order. 
Gilkey's approach to the original question involved two steps. He first studied conformal variations of the metric. This step is, of course, a logical one in our setting and it suffices to prove Theorem 0.3 . Conformal variations also reduce the proof of Theorem 0.2 to the special case in which the invariants are homogeneous of degree $m$ in the jets of the metric. Gilkey's second step involved stabilization; this was a reduction of the dimension $m$ carried out by considering product manifolds $N \times T^{1}$ endowed with their natural product metrics; since stabilization does not preserve conformal flatness, different techniques are required.

$\S 1$ is devoted to the proof of Theorem 0.1 . In $\S 2$, we use Gilkey's techniques of conformal variation to prove Theorem 0.3 and to reduce the proof of Theorem 0.2 to the special case in which the invariants are homogeneous of order $m$ in the jets of the metric. In $\S 3$, we use techniques of Anderson [1] and Olver [13] to further reduce the proof of Theorem 0.2 to the special case where the invariants involve only the jets of order at most 2 in the metric. In $\S 4$, we complete the proof by constructing an explicit basis for the space of second order invariants and by studying variational formulas.

In the original problem, one assumed that the invariant was unchanged under arbitrary variations in the metric. Here we show that the original conclusion remains valid under the weaker condition that it is unchanged by variations within the locally flat conformal structure.

We conclude the introduction by describing one of the problems which motivated us. We expect that our result will have applications in the extremal problem for the functional determinant of the conformal Laplacian

$$
L_{g}:=\Delta_{g}+\frac{m-2}{4(m-1)} \tau_{g}
$$

on the even dimensional spheres $S^{m}$. Here $g$ is a Riemannian metric which is locally conformal to the standard round metric $g_{0}$ and $\Delta_{g}$ (resp. $\tau_{g}$ ) is the Laplacian (resp. scalar curvature) of the metric $g$. The local conformality condition means that $g$ is of the form $g=e^{2 \omega} g_{0}$ for some smooth real function $\omega$ on $S^{m}$. We normalize the problem by assuming $\operatorname{vol}(g)=\operatorname{vol}\left(g_{0}\right)$. This extremal problem has been solved in the case of $S^{2}$ [14], $S^{4}$ [4], and $S^{6}$ [3]; in each case, the extremal values are attained exactly when $g$ is the pullback of $g_{0}$ by an element of the (finite-dimensional) conformal transformation group of $\left(S^{m}, g_{0}\right)$. The determinant quotient $\operatorname{det}\left(L_{g}\right) / \operatorname{det}\left(L_{g_{0}}\right)$ is related to the local invariant $a_{m}(x, L)$ from the asymptotics of the heat kernel trace $\operatorname{Tr}_{L^{2}}\left(e^{-t L}\right)$ as $t \downarrow 0^{+}$by a generalization of the Polyakov formula; we refer to [6] for a more complete discussion.

Thus analysis of the determinant quotient hinges on more or less explicit knowledge of the local invariant $a_{m}(x, L)$. By [5], $\int a_{m}(x, L)\left|d \nu_{g}\right|$ is a conformal invariant of compact Riemannian manifolds. On the other hand, the higher dimensional analysis of [3] hinges on a local scalar invariant $Q_{m}$ which takes the positive value $(m-1)$ ! on $\left(S^{m}, g_{0}\right)$, and for which $\int Q_{m}\left|d \nu_{g}\right|$ is a conformal invariant. Thus, in the locally conformally flat case, the result of this paper shows that

$$
a_{m}(x, L)=b_{1} E_{m}+\delta \eta_{1}=b_{2} Q_{m}+\delta \eta_{2},
$$

where the $b_{i}$ are real constants and the $\eta_{i}$ are one form valued local invariants. 
We can further rewrite things by applying a leading terms analysis as in [8]:

$$
\delta \eta_{2}=b_{3} \Delta^{(m-2) / 2} \tau+\delta \eta_{3} \text { and } Q_{m}=\widetilde{Q}_{m}+b_{4} \Delta^{(m-2) / 2} \tau,
$$

where $\delta \eta_{3}$ and $\widetilde{Q}_{m}$ have expressions as local invariants in which each term has at most $m-4$ covariant derivatives. Thus

$$
a_{m}(x, L)=b_{2} \widetilde{Q}_{m}+b_{5} \Delta^{(m-2) / 2} \tau+\delta \eta_{3} .
$$

Since one form valued local invariants are necessarily parallel on $\left(S^{m}, g_{0}\right)$ and since by [3] we know the zeta function of $L$ on $\left(S^{m}, g_{0}\right)$ explicitly, we know $b_{2}$ in principle. By [8], we also know $b_{5}$. But the terms in (0.14) with these coefficients produce the two terms in Polyakov type determinant quotient formula which are dominant in a certain precise sense having to do with inequalities of borderline Sobolev embedding and Moser Trudinger type.

It is a pleasant task to thank the referee for helpful comments and suggestions.

\section{LOCAL INVARIANTS OF LOCALLY CONFORMALLY FLAT MANIFOLDS}

We adopt the notational convention that Latin indices $i, j$, etc. range from 1 through $m$ and that they index a local orthonormal frame for the tangent bundle. Let $R_{i j k l}$ be the curvature tensor. We adopt the Einstein convention and sum over repeated indices. Let $\rho_{i j}:=R_{i k k j}$ and $\tau:=\rho_{i i}$ be the Ricci tensor and scalar curvature; with our sign convention, $R_{1221}=+1$ on the standard sphere in $\mathbf{R}^{3}$. Let

$$
J:=\frac{1}{2(m-1)} \tau \text { and } \quad V:=\frac{1}{(m-2)}(\rho-J g)
$$

be the normalized scalar curvature and the normalized Ricci tensor. Let $\delta_{i j}$ be the Kronecker symbol. Let

$$
C_{i j k l}:=-R_{i j k l}+\delta_{i l} V_{j k}-\delta_{i k} V_{j l}+\delta_{j k} V_{i l}-\delta_{j l} V_{i k}
$$

be the Weyl conformal tensor. If $m \geq 4$, then $M$ is locally conformally flat if and only if $C=0$; if $m=3$, then $C$ vanishes identically and $M$ is locally conformally flat if and only if the covariant derivative of $V$ is completely symmetric (i.e. the Cotton tensor

$$
V_{i j ; k}-V_{i k ; j}
$$

vanishes identically). We refer to Goldberg [10] for details.

Let $\mathscr{G}$ be the group of all germs of diffeomorphisms $\psi$ of $\mathbf{R}^{m}$ with

$$
\psi(0)=0, \quad d \psi(0)=I,
$$

and which are conformal; i.e. we assume that there exists a germ of a smooth function $h_{\psi}$ so that

$$
\psi^{*}(d x \circ d x)=e^{2 h_{\psi}} d x \circ d x .
$$

This is the structure group with which we shall be dealing. 
Lemma 1.1. (a) Given $\xi_{0} \in \mathbf{R}^{m}$, there exists $\psi \in \mathscr{G}$ so that $d h_{\psi}(0)=\xi_{0}$.

(b) Let $g$ be a locally conformally flat metric. At any point $x_{0} \in M$, we can choose coordinates so that $g=e^{2 h_{X}}(d x \circ d x)$ for $h_{X}\left(x_{0}\right)=0$ and $d h_{X}\left(x_{0}\right)=0$. Proof. Let $\Psi(x)=x /|x|^{2}$ be inversion about the unit sphere; we see that $\Psi$ is conformal by computing:

$$
\begin{aligned}
d \Psi= & \left(|x|^{-2} d x-2|x|^{-4}(x \cdot d x) x\right), \\
d \Psi \circ d \Psi= & |x|^{-4} d x \circ d x-4|x|^{-6}(x \cdot d x) \circ(x \cdot d x) \\
& +4|x|^{-8}|x|^{2}(x \cdot d x) \circ(x \cdot d x) \\
= & |x|^{-4} d x \circ d x .
\end{aligned}
$$

If $\xi_{0}=0$, we take $\psi$ to be the identity. If $\xi_{0} \neq 0$, let $x_{0}:=-2 \xi_{0}\left|\xi_{0}\right|^{-2}$; then $\xi_{0}=-2 x_{0}\left|x_{0}\right|^{-2}$. Let

$$
\psi(x):=d \Psi\left(x_{0}\right)^{-1}\left\{\Psi\left(x+x_{0}\right)-\Psi\left(x_{0}\right)\right\} ;
$$

$\psi(0)=0$ and $d \psi(0)=I$ so that $\psi \in \mathscr{G}$. The associated conformal factor is given by

$$
h_{\psi}=\frac{1}{2} \ln \left\{\left|x_{0}\right|^{4}\left|x+x_{0}\right|^{-4}\right\}
$$

and $d h_{\psi}(0)=-2 x_{0}\left|x_{0}\right|^{-2}=\xi_{0}$. This completes the proof of (a). If $g=$ $e^{h} d x \circ d x$, then

$$
\psi^{*}(g)=e^{h \circ \psi+h_{\psi}} .
$$

We choose $\psi$ so that $d h_{\psi}(0)+d h(0)=0$ and then rescale the resulting coordinate system to complete the proof of (b).

Proof of Theorem 0.1. Fix $x_{0} \in M$. Choose a conformal system of coordinates $X$ centered at $x_{0}$ so that

$$
g=e^{2 h_{x}}(d x \circ d x) .
$$

By Lemma 1.1, we may assume that $h_{X}\left(x_{0}\right)=0$ and $d h_{X}\left(x_{0}\right)=0$.

Let $0 \neq P \in \mathscr{P}_{m, n, p}$. Then $P(g)\left(x_{0}\right)$ is a polynomial in the jets of $h_{X}$ of order at least 2 . Let $\partial_{i}$ denote ordinary partial differentiation. The curvature tensor at $x_{0}$ takes the form

$$
R_{i j k l}=\delta_{j l} \partial_{i} \partial_{k} h+\delta_{i k} \partial_{j} \partial_{l} h-\delta_{i l} \partial_{j} \partial_{k} h-\delta_{j k} \partial_{i} \partial_{l} h
$$

Consequently $\rho_{i l}:=R_{i j j l}$ and $\tau$ are given at $x_{0}$ by

$$
\rho_{i l}=(2-m) \partial_{i} \partial_{l} h_{X}-\delta_{i l} \partial_{j} \partial_{j} h_{X} \text { and } \tau=2(1-m) \partial_{i} \partial_{i} h_{X}
$$

Let $V_{i j ; k}$ be the covariant derivative of the normalized Ricci tensor defined in (1.1). By (1.12)

$$
V_{i j}(g)\left(x_{0}\right)=-\partial_{i} \partial_{j} h\left(x_{0}\right) \text { and } V_{i j ; k}(g)\left(x_{0}\right)=-\partial_{i} \partial_{j} \partial_{k} h\left(x_{0}\right) .
$$

This shows that $V_{i j}$ and $V_{i j ; k}$ are symmetric tensors. Let $V^{(s)}:=V_{i_{1} \cdots i_{s}}$ be the complete symmetrization of the covariant derivative of $V$ of order $s-2$. Thus, for example,

$$
V_{i j k l}^{(2)}:=\frac{1}{4}\left(V_{i j k ; l}^{(1)}+V_{j k l ; i}^{(1)}+V_{k l i ; j}^{(1)}+V_{l i j ; k}^{(1)}\right) .
$$

We note that

$$
V_{i_{1} \cdots i_{s}}\left(x_{0}\right)=-\partial_{i_{1}} \cdots \partial_{i_{s}} h\left(x_{0}\right)+\mathscr{E}
$$


where the term $\mathscr{E}$ involves lower order jets of $h$ evaluated at $x_{0}$. Consequently, we can express the jets of $h$ in terms of these tensors; conversely these tensors are expressible in terms of the jets of $h$. Thus $P(g)=P\left(V_{i j}, V_{i j k}^{(1)}, \ldots\right)$ is a polynomial in these tensorial variables. As a result of (1.15), the variables $\left\{V_{i j}, V_{i j k}^{(1)}, \ldots\right\}$ are algebraically independent; there are no universal identities other than that of complete symmetry. It now follows that the forgetful functor from $\mathscr{Q}_{m, n, p}$ to $\mathscr{P}_{m, n, p}$ is an isomorphism which proves Theorem $0.1(\mathrm{a})$.

We form what is called a Weyl spanning set for $\mathscr{Q}_{m, 0, p}$ by considering all possible expressions which are homogeneous of degree $n$ where we contract indices in pairs; the spanning set is a basis for $p \leq 2 m$. For example, $\left\{\tau^{2}, \rho_{i j} \rho_{i j}, \tau ; j j\right\}$ is a basis for $\mathscr{Q}_{m, 0,4}$. Similarly, we form a Weyl spanning set for $\mathscr{Q}_{m, n, p}$ by alternating exactly $n$ indices and contracting the remaining indices in pairs. It now follows that $\mathscr{Q}_{m, n, p}=\{0\}$ if $n-p$ is odd. If $l$ is the number of variables in such a monomial, then $2 l \leq p$ since each variable has order 2 . Since the variables are completely symmetric, we can alternate at most one index per variable and thus $n \leq l$.

\section{CONFORMAL CHANGeS OF THE METRIC}

In this section, we will complete the proof of Theorem 0.3 and reduce the proof of Theorem 0.2 to the special case that $P \in \mathscr{P}_{m, 0, m}$. We begin by showing:

\section{Lemma 2.1.}

(a) Let $P \in \mathscr{P}_{m, 0}$ for $3 \leq m$. Assume that

$$
\int_{T^{m}} P(g)\left|d v_{g}\right|=0 \quad \forall g \in \mathscr{C} .
$$

Then there exists $P_{m} \in \mathscr{P}_{m, 0, m}$ and $Q \in \mathscr{P}_{m, 1}$ so that $P=P_{m}+\delta Q$.

(b) Let $P \in \mathscr{P}_{m, n}$ for $1 \leq n \leq m$. If $n<m$, assume that $d P=0$. If $n=m$, assume that

$$
\int_{T^{m}} P(g)=0 \quad \forall g \in \mathscr{C} .
$$

Then there exists $P_{n} \in \mathscr{P}_{m, n, n}$ and $Q \in \mathscr{P}_{m, n-1}$ so that $P=P_{n}+d Q$.

Proof. We first prove (a); we follow the treatment in [7]; see also [9, §2.9]. There exist invariant polynomials $P_{\nu}$ which are homogeneous of order $\nu$ in the jets of the metric so that

$$
\begin{gathered}
P=P_{0}+\cdots+P_{m}+\cdots+P_{n}, \\
P\left(c^{2} g\right)\left|d v_{c^{2} g}\right|=\sum_{\nu} c^{m-\nu} P_{\nu}(g)\left|d v_{g}\right| .
\end{gathered}
$$

Since each $P_{\nu}$ satisfies the hypothesis of Lemma 2.1(a) separately, we may assume without loss of generality that $P=P_{\nu}$ for some $\nu \neq m$.

We must now prove that $P=\delta Q$. Let $f \in C^{\infty}(M)$, let $\varepsilon$ be a real parameter, and let $g(\varepsilon, f)=e^{2 \varepsilon f} g$ be the conformally rescaled metric. We linearize the problem. Define $Q(f, g)$ by

$$
\left.\frac{d}{d \varepsilon}\right|_{\varepsilon=0}\left\{P(g(\varepsilon, f))\left|d v_{g(\varepsilon, f)}\right|\right\}=Q(f, g)\left|d v_{g}\right| .
$$


Since $Q(f, g)$ is linear in the jets of the function $f$, we expand

$$
Q(f, g)=\sum_{p} f_{; i_{1} \cdots i_{p}} Q_{i_{1} \cdots i_{p}}(g),
$$

where $Q_{i_{1} \cdots i_{p}}$ is symmetric in the indices $\left\{i_{1}, \ldots, i_{p}\right\}$. Let

$$
\begin{aligned}
S(g) & :=\sum_{p}(-1)^{p} Q_{i_{1} \cdots i_{p} ; i_{p} \cdots i_{1}}, \\
R(f, g) & :=\sum_{p}(-1)^{p}\left\{\sum_{0<j \leq p}(-1)^{j-1} f_{; i_{1} \cdots i_{j-1}} Q_{i_{1} \cdots i_{p} ; i_{p} \cdots i_{j+1}}\right\} e_{i_{j}} .
\end{aligned}
$$

Then $Q(f, g)=\delta R(f, g)+f S(g)$. We integrate by parts to see that if $g \in \mathscr{C}$, then

$$
0=\left.\frac{d}{d \varepsilon}\right|_{\varepsilon=0} \int_{T^{m}} P(g(\varepsilon, f))\left|d v_{g}\right|=\int_{T^{m}} f S(g)\left|d v_{g}\right| .
$$

Since this holds for all $f \in C^{\infty}\left(T^{m}\right), S(g) \equiv 0$. Because any locally conformally flat metric is locally isometric to $g \in \mathscr{C}$ and because the invariants are locally defined, $S$ must vanish identically. Thus

$$
Q(f, g)=\delta R(f, g) \text {. }
$$

We now set $f=1$ and use (2.1) to complete the proof of (a) by checking:

$$
\begin{gathered}
\left.\frac{d}{d \varepsilon}\right|_{\varepsilon=0}\left\{P(g(\varepsilon, f))\left|d v_{g(\varepsilon, f)}\right|_{f=1}\right\}=(m-\nu) P(g)\left|d v_{g}\right|, \\
P(g)=(m-\nu)^{-1} Q(1, g)=\delta\left\{(m-\nu)^{-1} R(1, g)\right\} .
\end{gathered}
$$

We shall omit the proof of (b) since it follows directly from the arguments given in $[9, \S 2.9 .3]$ in the Riemannian case and is, in any event, very similar to the proof of $(a)$.

Proof of Theorem 0.3. We apply Lemma 2.1. Let $P \in \mathscr{P}_{m, n}$ satisfy the hypothesis of Theorem 0.3. By (b), there exists $P_{n} \in \mathscr{P}_{m, n, n}$ and $Q \in \mathscr{P}_{m, n-1}$ so that $P=P_{n}+d Q$. By Theorem $0.1, P_{n}=0$.

Suppose that $P \in \mathscr{P}_{m, 0}$ satisfies the hypothesis of Theorem 0.2. By Lemma 2.1(a), there exist $Q \in \mathscr{P}_{m, 1}$ and $P_{m} \in \mathscr{P}_{m, 0, m}$ so that

$$
P=P_{m}+\delta Q \text {. }
$$

This reduces the proof of Theorem 0.2 to the special case $P \in \mathscr{P}_{m, 0, m}$. If $m$ is odd, then $P=0$ which completes the proof in this case. We therefore assume $m$ even henceforth.

\section{ELIMINATION OF THE HIGHER ORDER JETS}

This section is devoted to the proof of the following technical result which eliminates the higher order jets from consideration. 
Lemma 3.1. Let $P \in \mathscr{P}_{m, 0, m}$ for $3 \leq m$. Assume that

$$
\int_{T^{m}} P(g)\left|d v_{g}\right|=0 \quad \forall g \in \mathscr{C} .
$$

Then there exist a polynomial $Q \in \mathscr{P}_{m, 1, m-1}$ and a polynomial $P_{1}=P_{1}\left(V_{i j}\right) \in$ $\mathscr{P}_{m, 0, m}$ so that $P=P_{1}+\delta Q$.

We begin the proof of Lemma 3.1 with a series of technical lemmas. We work on the torus $T^{m}$ with the flat metric for the moment. If $h \in C^{\infty}\left(T^{m}\right)$, let $\mathscr{S}^{r} h$ be the symmetric tensor given by the jets of $h$ of order $r$. Let $\mathscr{A}_{m, n}(v)$ be the vector space of all polynomials

$$
P(v ; h):=\sum_{|I|=n} P_{I}\left(v ; h, \mathscr{S}_{h}, \ldots, \mathscr{S}^{n} h\right) d x^{I}
$$

which are $n$ form valued and which depend polynomially on certain auxiliary variables $v$ and on the jets of $h$ up to order $n$. We shall need these auxiliary parameters in the proof of Lemma 3.2. We do not impose any condition of invariance on $P$. Let $\mathscr{A}_{m, n, p}(v)$ be the subspace of polynomials which are homogeneous of order $p>0$ in the derivatives of $h$.

If $P \in \mathscr{A}_{m, n, p}(v)$, define

$$
\mathscr{W}(P)(v ; h ; t):=\sum_{|I|=n} P_{I}\left(v ; h, \mathscr{S}_{h}, \mathscr{S}^{2} h, t \mathscr{S}^{3} h, \ldots, t^{n-2} \mathscr{S}^{n} h\right) d x^{I} .
$$

Let $\omega(P)$ be the degree of $\mathscr{W}(P)$ in the parameter $t ; \omega(P)>0$ if and only if $P$ involves jets of order at least 3 . We differentiate $P$ with respect to $h$ and hold the variables $v$ fixed to define

$$
d P \in \mathscr{A}_{m, n+1, p+1}(v) \text { and } \delta P \in \mathscr{A}_{m, n-1, p+1}(v) .
$$

We use arguments of Anderson [1] and Olver [13] in the proof of the following lemma.

Lemma 3.2. Let $P \in \mathscr{A}_{m, n, p}(v)$ for $1 \leq p \leq n$. If $n<m$, assume that $d P=0$. If $n=m$, assume that

$$
\int_{T^{m}} P(v ; h)=0 \quad \forall h \in C^{\infty}\left(T^{m}\right) .
$$

Then there exists $Q \in \mathscr{A}_{m, n-1, p-1}(v)$ so that $d Q=P$ and

$$
\omega(Q) \leq \max (0, \omega(P)-1) .
$$

Proof. Suppose first that $n=1$. Then $P$ is linear in the 1-jets of $h$ so $\omega(P)=$ 0 . We must construct $Q$ so that $\omega(Q)=0$. Let

$$
P=\sum_{i, j, k} a_{i j k}(v) \partial_{j} h^{i+1} \cdot d x^{k}
$$

We compute that

$$
0=d P=\sum_{i, j, k, l} a_{i j k}(v) \partial_{j} \partial_{l} h^{i+1} \cdot d x^{l} \wedge d x^{k} .
$$


Let $j \neq k$. Since only $a_{i j k}(v)$ gives rise to $\partial_{j}^{2} h^{i+1} d x^{j} \wedge d x^{k}, a_{i j k}=0$. Therefore $P=\sum_{i, j} a_{i j}(v) \partial_{j}\left\{h^{i+1}\right\} d x^{j}$, and

$$
d P=\sum_{i, j, k}\left(a_{i j}-a_{i k}\right) \partial_{k} \partial_{j} h^{i+1} \cdot d x^{k} \wedge d x^{j} .
$$

This shows that $a_{i j}=a_{i k}=a_{i}$. The lemma now follows for $n=1$ since

$$
P=\sum_{i} a_{i} \partial_{j} h^{i+1} \cdot d x^{j}=d\left\{\sum_{i} a_{i}(i+1)^{-1} h^{i+1}\right\} .
$$

We proceed by induction on $n$ and assume that the lemma has been proved for forms of lower degree. We use the argument of Lemma 2.1 to choose a polynomial $Q \in \mathscr{A}_{m, n-1, p-1}(v)$ with $\omega(Q)$ minimal such that $d Q=P$. Let $a=\omega(Q)$. Suppose that the lemma fails so that

$$
a \geq \omega(P) \text { and } a>0 .
$$

Let $Q_{a}$ be the sum of the monomials $A$ in $Q$ with $\omega(A)=a$. Decompose

$$
d Q=d_{1} Q+d_{2} Q,
$$

where $d_{1}$ differentiates $\{h, \mathscr{S} h\}$ and $d_{2}$ differentiates the remaining jets of $h$. We expand

$$
d Q=d_{1} Q+d_{2} Q .
$$

Expand $\mathscr{W}(Q)=t^{a} Q_{a}+\cdots+Q_{0}$. It is then immediate that

$$
\begin{aligned}
O\left(t^{a}\right) & =\mathscr{W}(P)=\mathscr{W}(d Q)=d_{1} \mathscr{W}(Q)+t d_{2} \mathscr{W}(Q) \\
& =t^{a+1} d_{2}\left(Q_{a}\right)+O\left(t^{a}\right) .
\end{aligned}
$$

This shows that $d_{2} Q_{a}=0$. We next freeze the value of $h$ and of $\mathscr{S} h$. Introduce an auxiliary variable $\tilde{v}=\left(\tilde{v}_{0}, \xi\right)$ for $\tilde{v}_{0} \in \mathbf{R}$ and $\xi \in \mathbf{R}^{m}$ and define $\widetilde{Q}_{a}=\widetilde{Q}_{a}(v ; \tilde{v} ; h)$ by

$$
\widetilde{Q}_{a}(v ; \tilde{v} ; h):=Q_{a}\left(v ; \tilde{v}_{0}, \xi, \mathscr{S}^{2} h, \ldots\right) .
$$

Then $d \tilde{Q}_{a}=d_{2} Q_{a}=0$. Since $\omega\left(Q_{a}\right)>0, Q_{a}$ involves derivatives of order at least 3. These variables are not frozen so $\widetilde{Q}_{a}$ is homogeneous of degree $\tilde{p}$ where $3 \leq \tilde{p} \leq n-1$. We may therefore apply the induction argument to find

$$
\begin{aligned}
& \tilde{R} \in \mathscr{A}_{m, n-2, \tilde{p}-1}(v ; \tilde{v} ; h) \text { so that } \\
& d \tilde{R}\left(v ; \tilde{v} ; \mathscr{S} h, \mathscr{S}^{2} h, \ldots\right)=\widetilde{Q}_{a}(v ; \tilde{v} ; h) \text { and } \omega(\widetilde{R}) \leq a-1 .
\end{aligned}
$$

We now unfreeze the coefficients and define

$$
R(v ; h):=\widetilde{R}\left(v ; h, \mathscr{S}_{h} ; h, \mathscr{S}_{h}, \mathscr{S}^{2} h, \ldots\right) .
$$

Differentiating $R$ with respect to $h$ or $\mathscr{S} h$ does not change the weight $\omega$ since the derivatives of these variables can be of degree at most 2 . Consequently

$$
d R(v ; h)=Q_{a}+\mathscr{E},
$$

where the error $\mathscr{E}$ satisfies $\omega(\mathscr{E}) \leq a-1$. Let $Q_{1}=Q-d R$. Then $d Q_{1}=P$ and $\omega\left(Q_{1}\right)<a$. This contradicts the minimality of $\omega(Q)$.

We drop the auxiliary parameters $v$ henceforth; they were only required for the proof of Lemma 3.2 where we froze and then unfroze the 0 and 1 jets of $h$. We continue with another technical lemma. Let $|d x|$ be the standard measure on the torus. 
Lemma 3.3. Let $P \in \mathscr{A}_{m, m, m}$ with $P(h)=P\left(\mathscr{S}^{2} h, \ldots, \mathscr{S}^{m} h\right)$. Assume that

$$
\int_{T^{m}} P(h)|d x|=0 \quad \forall h \in C^{\infty}\left(T^{m}\right) .
$$

Then there exists $Q \in \mathscr{A}_{m, m-1, m-1}$ with $Q(h)=Q\left(\mathscr{S}^{2} h, \ldots, \mathscr{S}^{m} h\right)$ so that

$$
P-\delta Q=R\left(\mathscr{S}^{2} h\right) \text {. }
$$

Proof. Let the length $l(A)$ of a monomial $A$ be the degree of $A$ as a polynomial in $h$ and its derivatives. This means that

$$
A\left(s h, s \mathscr{S}_{h}, \ldots, s \mathscr{S}^{r} h\right)=s^{l(A)} A\left(h, \mathscr{S}_{h}, \ldots, \mathscr{S}^{r} h\right) .
$$

We decompose $\mathscr{A}_{m, n, p}=\bigoplus_{l} \mathscr{A}_{m, n, p}^{l}$ where the monomials of any polynomial in $\mathscr{A}_{m, n, p}^{l}$ are all of length $l$. We use (3.16) to see that

$$
d: \mathscr{A}_{m, n, p}^{l} \rightarrow \mathscr{A}_{m, n+1, p+1}^{l} \text {. }
$$

Consequently exterior differentiation preserves this grading. We decompose

$$
P=P_{1}+\cdots+P_{\nu} \quad \text { where } P_{l} \in \mathscr{A}_{m, m, m}^{l} \text {. }
$$

Since each $P_{l}$ satisfies the conditions of the lemma separately, we may assume without loss of generality that $P=P_{l}$ henceforth.

Let $\psi(A, \nu)$ be the number of variables with exactly $\nu$ derivatives which appear in $A$ when counted with multiplicity. In other words

$$
\begin{aligned}
& A\left(h, \ldots, \mathscr{S}^{\nu-1} h, s^{\nu} h, \mathscr{S}^{\nu+1} h, \ldots\right) \\
& \quad=s^{\psi(A, \nu)} A\left(h, \ldots, \mathscr{S}^{\nu-1} h, \mathscr{S}^{\nu} h, \ldots\right) .
\end{aligned}
$$

Let $A$ be homogeneous of order $n$ in the derivatives of the metric. Then

$$
\begin{aligned}
l(A) & =\psi(A, 0)+\psi(A, 1)+\cdots, \\
n & =\psi(A, 1)+2 \psi(A, 2)+\cdots .
\end{aligned}
$$

Let $P$ satisfy the hypothesis of the lemma and let $A$ be a monomial of $P$. Then only jets of order at least 2 appear in $A$ and $A$ is homogeneous of order $m$ in the derivatives of the metric. Thus

$$
\begin{aligned}
m & =\psi(A, 2) \cdot 2+\psi(A, 3) \cdot 3+\cdots+\psi(A, m) \cdot m \\
& =2 l(A)+\psi(A, 3)+\psi(A, 4) \cdot 2+\cdots+\psi(A, m) \cdot(m-2) \\
& =2 l(A)+\omega(A) .
\end{aligned}
$$

This shows that

$$
\omega(A)=m-2 l
$$

for every monomial $A$ of $P$. If $2 l=m$, then $\omega(A)=0$ so that $A$ only involves the 2-jets of $h$; the lemma follows. We therefore assume $2 l<m$ so that $\omega(A)>0$ for every monomial $A$ of $P$. that

We apply Lemma 3.2 to choose $Q \in \mathscr{A}_{m, m-1, m-1}$ so that $d Q=P$ and so

$$
\omega(Q) \leq \omega(P)-1 .
$$

We may assume without loss of generality that $Q \in \mathscr{A}_{m, m-1, m-1}^{l}$. Let $A$ be a monomial of $Q$. Then

$$
\begin{aligned}
m-1 & =0 \cdot \psi(A, 0)+1 \cdot \psi(A, 1)+2 \cdot \psi(A, 2)+3 \cdot \psi(A, 3)+\cdots \\
& \leq 2 l+\psi(A, 3)+2 \cdot \psi(A, 4)+\cdots=2 l+\omega(A) .
\end{aligned}
$$


Consequently, $\omega(A) \geq m-2 l-1=\omega(P)-1$. Since $\omega(A) \leq \omega(P)-1$, equality holds in (3.24). This shows that

$$
\psi(A, 0)=\psi(A, 1)=0 .
$$

We use Lemmas 3.2 and 3.3 to complete the proof of Lemma 3.1. Let the length $\mathscr{L}(A)$ of a monomial $A\left(V, V^{(1)}, \ldots, V^{(s)}\right)$ be the degree of $A$ as a polynomial in the tensor $V$ and its symmetrized covariant derivatives. This defines a grading

$$
\mathscr{P}_{m, n, p}=\bigoplus_{\mathscr{L}} \mathscr{P}_{m, n, p}^{\mathscr{L}} ; \quad d \mathscr{P}_{m, n, p}^{\mathscr{L}} \subseteq \bigoplus_{\nu \geq \mathscr{L}} \mathscr{P}_{m, n+1, p+1}^{\nu} .
$$

Let $P \in \mathscr{P}_{m, 0, m}$ for $3 \leq m$. Assume that

$$
\int_{T^{m}} P(g)\left|d v_{g}\right|=0 \quad \forall g \in \mathscr{C} .
$$

Decompose $P=P_{0}+P_{1}+\cdots$, where $P_{\nu} \in \mathscr{P}_{m, 0, m}^{\nu}$. Choose $\mathscr{L}$ minimal with $P_{\mathscr{L}} \neq 0$ and let $\mathscr{L}(P)=\mathscr{L}$. Choose $Q \in \mathscr{P}_{m, 1, m-1}$ so that $\mathscr{L}(P-\delta Q)$ is maximal; we replace $P$ by $P-\delta Q$ to simplify the notation involved. If $2 \mathscr{L}=m$, then $P=P\left(V_{i j}\right)$ so we assume that $2 \mathscr{L}<m$. Thus $P$ involves some variable of order at least 3.

Let $\mathscr{E}$ denote a generic polynomial which is the sum of monomials of lengths greater than $\mathscr{L}$. Choose a local conformal coordinate system so that $g=$ $e^{2 h} d x \circ d x$. Then

$$
V_{i_{1} \cdots i_{\nu}}=e^{-\nu h} \partial_{i_{1}} \cdots \partial_{i_{\nu}} h+\mathscr{E} .
$$

Let $\star$ be the Hodge operator, and let $d x$ be the standard volume form on $\mathbf{R}^{m}$. We dualize to expand

$$
\star P(h)=\left\{P_{\mathscr{L}}\left(\mathscr{S}^{2} h, \mathscr{S}^{3} h, \ldots\right)+\mathscr{E}\right\} d x .
$$

The crucial point is that the factors of $e^{-h}$ have disappeared when we applied the Hodge operator so that $\star P(h)$ is polynomial. By replacing $h$ by $s h$, we see that

$$
\begin{aligned}
0 & =\int_{T^{m}} \star P(s h) \\
& =s^{\mathscr{L}} \int_{T^{m}} P_{\mathscr{L}}\left(\mathscr{S}^{2} h, \mathscr{S}^{3} h, \ldots\right)|d x|+O\left(s^{\mathscr{L}+1}\right) .
\end{aligned}
$$

Consequently

$$
\int_{T^{m}} P_{\mathscr{L}}\left(\mathscr{S}^{2} h, \mathscr{S}^{3} h, \ldots\right) d x=0 \quad \forall h \in C^{\infty}\left(T^{m}\right) .
$$

We may therefore apply Lemma 3.3 to find a polynomial $Q\left(\mathscr{S}^{2} h, \mathscr{S}^{3} h, \ldots\right)$ so that

$$
d Q\left(\mathscr{S}^{2} h, \ldots\right)=P_{\mathscr{L}}\left(\mathscr{S}^{2} h, \ldots\right) .
$$

We now replace $\mathscr{S}^{2} h$ by $V_{i j}, \mathscr{S}^{3} h$ by $V_{i j k}$, and so forth to see that

$$
d Q\left(V_{i j}, V_{i j k}, \ldots\right)=P_{\mathscr{L}}\left(V_{i j}, V_{i j k}, \ldots\right)+\mathscr{E} \text {. }
$$


Dually, this yields the formula

$$
P-\delta\left(\star Q\left(V_{i j}, V_{i j k}, \ldots\right)\right)=\mathscr{E} .
$$

The polynomial $\star Q$ need not be invariant. However, we may integrate over the structure group $O(m)$ to create $Q$ satisfying (3.34) which is $O(m)$ invariant. Since $\mathscr{L}(\mathscr{E})>\mathscr{L}$, this contradicts the choice of $P$ and completes the proof of Lemma 3.1.

\section{INVARIANTS IN THE 2-JETS OF THE METRIC}

Let

$$
\begin{aligned}
& \mathscr{K}:=\left\{P \in \mathscr{P}_{m, 0, m}: \int_{T^{m}} P(g)\left|d v_{g}\right|=0 \forall g \in \mathscr{C}\right\}, \\
& \mathscr{B}_{2}:=\left\{P \in \mathscr{P}_{m, 0, m}: P=P\left(V_{i j}\right)\right\} .
\end{aligned}
$$

We have reduced the proof of Theorem 0.2 to the following technical result.

Lemma 4.1. Let $P \in \mathscr{K} \cap \mathscr{B}_{2}$. Then $P=c E_{m}$.

Proof. Since $E_{m} \in \mathscr{K} \cap \mathscr{B}_{2}$, it suffices to show that $\operatorname{dim}\left(\mathscr{K} \cap \mathscr{B}_{2}\right) \leq 1$. Let $1 \leq s<\infty$ and $\vec{s}=\left(s_{1}, s_{2}, \ldots\right)$. We define:

$$
\begin{gathered}
P_{s}:=\operatorname{Tr}\left(V^{s}\right)=V_{i_{1} i_{2}} V_{i_{2} i_{3}} \cdots V_{i_{s} i_{1}}, \\
|\vec{s}|:=s_{1}+2 s_{2}+\cdots, \quad \text { and } P_{s}:=P_{1}^{s_{1}} P_{2}^{s_{2}} \cdots .
\end{gathered}
$$

$P_{\vec{s}}$ is a polynomial which is of order $2|\vec{s}|$ in the jets of the metric; $P_{1}$ is a positive multiple of the scalar curvature $\tau$. The discussion of $\S 2$ shows that $\left\{P_{\vec{s}}\right\}_{|\vec{s}|=m}$ is a basis for $\mathscr{B}_{2}$. Expand $P \in \mathscr{B}_{2} \cap \mathscr{K}$ in the form

$$
P=\sum_{|\vec{s}|=m} c\left(P_{\vec{s}}, P\right) \cdot P_{\vec{s}}
$$

the linear functions $c\left(P_{\vec{s}}, P\right)$ are the dual coordinates on $\mathscr{B}_{2}$. We will show that $c\left(P_{1}^{m}, P\right)=0$ and $P \in \mathscr{B}_{2} \cap \mathscr{K}$ implies $P=0$; thus the linear functional $c\left(P_{1}^{m}, \cdot\right)$ separates points of $\mathscr{B}_{2} \cap \mathscr{K}$ and consequently $\operatorname{dim}\left(\mathscr{B}_{2} \cap \mathscr{K}\right) \leq 1$.

Let $f \in C^{\infty}\left(T^{m}\right)$ have support in a small neighborhood of the basepoint $x_{0} \in T^{m}$. Consider the variation

$$
g(\varepsilon):=e^{2 \varepsilon f} g .
$$

Let $e_{i}(\varepsilon)=e^{-\varepsilon f}$, where $e_{i}$ is a local orthonormal frame with respect to the metric $g$. We then compute that

$$
\left.\frac{d}{d \varepsilon}\right|_{\varepsilon=0} V_{i j}(g(\varepsilon))=-f_{; i j}-2 f V_{i j} .
$$

We integrate by parts to define a linear map

$$
\mathscr{F}: \mathscr{P}_{m, 0, n} \rightarrow \mathscr{P}_{m, 0, n}
$$

by the identity

$$
\left.\frac{d}{d \varepsilon}\right|_{\varepsilon=0} \int_{T^{m}} Q\left(g_{\varepsilon}\right)\left|d v_{g(\varepsilon)}\right|=\int_{T^{m}} f \cdot \mathscr{F}(Q)(g)\left|d v_{g}\right| \quad \forall Q \in \mathscr{P}_{m, 0, n}
$$


It is immediate that if $P \in \mathscr{K}$, then $\mathscr{F}(P)=0$ and therefore

$$
\sum_{\vec{s}} c\left(P_{\vec{s}}, P\right) \mathscr{F}\left(P_{\vec{s}}\right)=0 .
$$

We introduce an equivalence relation $\equiv$ on $\mathscr{F}\left(\mathscr{B}_{2}\right)$ by ignoring monomials which contain the expressions

$$
\left\{V_{i j k l}, V_{i i j}, V_{i j k}\right\},
$$

where $\{i, j, k, l\}$ are distinct indices; thus we are only interested in monomials which contain a variable of order 4 where an index repeats.

We note

$$
\left.\frac{d}{d \varepsilon}\right|_{\varepsilon=0} P_{s}=-s f_{; k_{1} k_{2}} V_{k_{2} k_{3}} \cdots V_{k_{s} k_{1}}-2 s f P_{s} .
$$

We will ignore the term $-2 s f P_{s}$ since it leads to terms $\equiv 0$. If $s>1$, then $k_{1} \neq k_{2}$ and

$$
\begin{aligned}
\int_{T^{m}}\left(\left.\frac{d}{d \varepsilon}\right|_{\varepsilon=0} P_{s}\right) P_{\vec{t}}\left|d v_{g}\right| & =-2 s \int_{T^{m}} f \cdot\left(V_{k_{2} k_{3} ; k_{1} k_{2}} V_{k_{2} k_{3}} \cdots V_{k_{s} k_{1}} P_{\vec{t}}+A\right) \\
& =\frac{-2 s}{s-1} \int_{T^{m}} f \cdot\left(P_{s-1 ; k k} P_{\vec{t}}+A_{1}\right)
\end{aligned}
$$

where $A_{1} \equiv A \equiv 0$; the covariant derivatives $k_{1} k_{2}$ can only attach themselves to either $V_{k_{2} k_{3}}$ or $V_{k_{1} k_{s}}$. On the other hand, if $s=1$, then $k_{1}=k_{2}$ and these derivatives can attach themselves as a unit to any $V_{i i}$ or $V_{i j}$ variable. More precisely, if $\phi(\nu)=2 \frac{\nu}{\nu-1}$, then

$$
\begin{aligned}
\mathscr{F}\left(P_{\breve{s}}\right) \equiv & -i_{1}\left(i_{1}-1\right) P_{1}^{i_{1}-2} P_{1 ; i i} P_{2}^{i_{2}} \ldots \\
& -i_{1} P_{1}^{i_{1}-1} \sum_{\nu \geq 2} i_{\nu} P_{2}^{i_{2}-1} \cdots P_{\nu-1}^{i_{\nu-1}} P_{\nu}^{i_{\nu}-1}\left(P_{\nu}\right)_{; i i} P_{\nu+1}^{i_{\nu+1}} \cdots \\
& -\sum_{\nu \geq 2} i_{\nu} \phi(\nu) P_{1}^{i_{1}} P_{2}^{i_{2}} \cdots P_{\nu-1}^{i_{\nu-1}}\left(P_{\nu-1 ; i i}\right) P_{\nu}^{i_{\nu}-1} P_{\nu+1}^{i_{\nu+1}} \cdots .
\end{aligned}
$$

Let $A=P_{1}^{i_{1}} \ldots P_{\nu}^{i_{\nu}} \ldots$ be a monomial of $P$. Choose $A$ so that $i_{1}$ is maximal; by hypothesis $i_{\nu}>0$ for some $\nu>1$ since $P_{1}^{m}$ is not a monomial of $P$. Then

$$
\tilde{A}=P_{1}^{i_{1}} \cdots P_{\nu-1}^{i_{\nu-1}} P_{\nu-1 ; i i} P_{\nu}^{i_{\nu}-1} \cdots
$$

is a monomial of $\mathscr{F}(A)$. Since the coefficient of $\tilde{A}$ in $\mathscr{F}(P)$ is zero, $\tilde{A}$ must be a monomial of $\mathscr{F}(B)$ for some other monomial $B$ of $P$. But $\tilde{A}$ is created either through the variation of a $P_{\mu}$ term for $\mu>1$ or through the variation of a $P_{1}$ term. In the former instance, $B=A$. In the latter instance, $P_{1}^{i_{1}+1}$ divides $B$ which contradicts the maximality of $A$.

\section{BIBLIOGRAPHY}

1. I. Anderson, The variational bicomplex, Academic Press, Boston, MA (to appear).

2. A. Avez, Characteristic classes and Weyl tensor. applications to general relativity, Proc. Nat. Acad. Sci. U.S.A. 66 (1970), 265-268. 
3. T. Branson, Sharp inequalities, the functional determinant, and the complementary series, Trans. Amer. Math. Soc. (to appear).

4. T. Branson, S.-Y. A. Chang, and P. Yang, Estimates and extremals for zeta function determinants on four manifolds, Comm. Math. Phys. 149 (1992), 241-262.

5. T. Branson and B. Orsted, Conformal indices of Riemannian manifolds, Compositio Math. 60 (1986), 261-293.

6. $\ldots$ Conformal geometry and global invariants, Differential Geom. Appl. 1 (1991), 279308.

7. P. Gilkey, Local invariants of an embedded Riemannian manifold, Ann. of Math. 102 (1975), 187-203.

8. L Leading terms in the asymptotics of the heat equation, Contemp. Math. 73 (1988), 79-85.

9. __ Invariance theory, the heat equation, and the Atiyah Singer index theorem, 2nd ed., CRC Press (to appear).

10. S. Goldberg, Curvature and homology, Pure Appl. Math., vol. 11, Academic Press, 1962.

11. J. Lee and T. Parker, The Yamabe problem, Bull. Amer. Math. Soc. (N.S.) 17 (1987), 37-91.

12. E. Miller, Ph.D. thesis, M.I.T.

13. P. J. Olver, Conservation laws and null divergences, Math. Proc. Cambridge Philos. Soc. 94 (1983), 529-540.

14. E. Onofri, On the positivity of the effective action in a theory of random surfaces, Comm. Math. Phys. 86 (1982), 321-326.

15. H. Weyl, The classical groups, Princeton Univ. Press, Princeton, NJ, 1946.

Department of Mathematics, University of Iowa, Iowa City, Iowa 52242

E-mail address: bransonemath.uiowa.edu

Department of Mathematics, University of OREgon, Eugene, OREgon 97403

E-mail address: gilkey@math.uoregon.edu

Department of Mathematics, Oregon State University, Corvallis, Oregon 97331

E-mail address: juha@math.orst.edu 